susceptibility to severe infections with distinctive susceptibility to various types of pathogens, like mycobacterium bovis in BCG (Bacille Calmette-Guérin) vaccine.

Methods We conducted retrospective study (demographics, investigations and treatments) over 5-years period, between 2007 and 2011, at King Abdulaziz Medical City-WR, Saudi Arabia for patients diagnosed with disseminated BCGitis.

Results Out of 16 susceptible immunodeficiency patients given BCG vaccine at birth, 6 patients (37.5\%); one chronic granulomatous disease, one ectodermal dysplasia/cytokines deficiency and 4-severe combined immunodeficiency were diagnosed to have disseminated BCGitis. The average age at time of diagnosis was $10^{1 / 2}-$ months (range: 3 to 30 months), one patient was diagnosed postbone marrow transplantation. The diagnosis confirmed by microbiologic stain and TB cultures from lungs, gastrointestinal tract and skin biopsies. All patients received 4 anti-tuberculous (TB) drugs, except one patient still on five anti-TB and IFN- $\gamma$ treatments for a year. In compression to studies from nonindustrial countries (their survival rates ranges: zero to $50 \%$ ), we achieved an excellent survival rate (100\%). However, one patient developed chronic lung disease.

Conclusion Our results (100\% survival rate) highlight the importance of high index of suspicion, early diagnosis of PID and BCGitis and promote intervention with anti-TB drugs. However, because of prolonged anti-TB therapies and high fatality rates of BCGitis reported from nonindustrial countries where also BCG vaccine is compulsory at birth, we recommend suspending BCG vaccine for susceptible PID newborns till such diagnosis ruled out.

\section{AN EXPERIENCE OF USAGE OF ACELLULAR DTAP VACCINE IN CHILDREN OVER 4 YEARS OLD}

doi:10.1136/archdischild-2012-302724.1827

A Gayvoronskaya, M Galitskaya, T Grechukha. Scientific Centre of Children's Health, Moscow, Russia

Actuality: Pertussis is a high contagious bacterial infection with an air-drop way of transmission, remains a serious problem as in Russia, as all over the world. The only reliable way of prevention is immunization. The booster dose against pertussis injects at the age of one and a half years by the integral cellular DTP vaccine. In connection with the decrease of intensiry of immunity in 4-6 years after it, especially among children of school age, teenagers and adults, pertussis grows in these age groups. It is necessary to apply less reaktogenny acellular DTaP vaccine.

Purpose To estimate the shipping of $\mathrm{DTaP}$ vaccine in children over 4 years old as healthy, as with different deviations in a state of health.

Materials and Methods 83 children were immunized at the age of 4-12 years old: 29 health children and 54 children with various chronic diseases. The shipping of immunization was analysed.

Results Side effects, which have developed in first 3 days after immunization, have been noted at $27.7 \%$ of children with allergic pathology. All of them were moderate degree of expressiveness (temperature not higher than 38.5 C, hyperemia no more than 4-5 $\mathrm{sm})$. Noone strong reactions were fixed.

Conclusion DTaP vaccine showed well safety profile, side effects in children with allergic pathology were moderate, had a shirt-term. $\mathrm{DTaP}$ vaccine is recommended as a booster dose in children at the age of 6-7 years old for inclusion to the National Calendar of inoculation of Russia.

\section{PNEUMOCOCCAL DISEASES IN CHINA, OUR RECENT} STUDIES

doi:10.1136/archdischild-2012-302724.1828

\section{Y Yang. Beijing Children's Hospital, Capital Medical University, Beijing, China}

Our reviewed data demonstrated that Streptococcus pneumoniae (S. pneumoniae) is an important pathogen and death of pyogenic meningitis, pneumonia, and other infectious diseases in children. The distribution of serotypes of $S$. pneumoniae showed great diversity in several studies. The penicillin nonsusceptibility rates demonstrated an increase over time in China. The prevalence of resistance to erythromycin was very high. A total of 202 paraffinembedded lung autopsy tissues of children aged 1 month to 5 years old who died of CAP were selected at random from a hospital. Conventional PCR, southern blotting and ISPCR were used to detect $S$. pneumoniae in lung tissues for a mouse pneumonia model and in 202 autopsy samples from fatal childhood CAP cases, 1 month to 5 years old, between 1953-2002. Southern blotting and ISPCR detected S. pneumoniae in 107/202 (53.0\%) and 106/202 $(52.5 \%)$ human samples respectively. A combined total of $116 / 202$ (57.4\%) samples were found to be positive by both methods. There is evidence that $\mathrm{S}$. pneumoniae was an important cause of fatal childhood CAP in China, as elsewhere. Recently a total of 171 Streptococcus pneumoniae isolates causing invasive disease were isolated from Chinese children. The serotype distribution and antimicrobial resistance were tested. The results suggested that the 7-valent pneumococcal conjugate vaccine has a preventive effect among children and that there should be long-term surveillance for serotype 19A. Pneumococcal disease burden in China should be undertaken in the future to provide evidence and guidance to the use of vaccine and antibiotics in $S$. pmeumoniae infections.

\section{SEVERE FORMS OF OSTEOGENESIS IMPER.FECTA (OI) IN INFANTS AND THE ROLE OF RESPIRATORY SYNCITIAL VIRUS (RSV) IMIMUNOPROPHYLAXIS WITH PALIVIZUMAB}

doi:10.1136/archdischild-2012-302724.1829

RK Philip, S Qadri. Paediatrics, University Hospital Limerick, Limerick, Ireland

Background and Aims Respiratory illnesses could be serious, even life threatening among infants with severe forms of osteogenesis imperfecta (OI). Respiratory syncitial virus (RSV) bronchiolitis could deteriorate the respiratory status of infants with severe OI increasing length of stay (LOS) and often needing paediatric high dependency unit (PHDU) or paediatric intensive care unit (PICU) care. Immunoprophylaxis using Palivizumab - monoclonal antibody to RSV F protein could prevent RSV bronchiolitis for severe OI infants. We aimed to audit our local practice and national data.

Methods We conducted a retrospective chart review of infants with severe OI in the Mid-West of Ireland from 2005 to 2010, all of whom received Palivizumab in addition to the standard care for OI with early bisphosphonates. From national computerised Hospital In-Patient Enquiry (HIPE) data information on all infants in Ireland with OI admission and RSV positive bronchiolitis was tabulated. LOS and bed days use nationally were determined. Information of immunoprophylaxis for national OI cohort was obtained from the national provider of Palivizumab. Hospital audit committee approval was sought.

Results No infant in the Mid-West of Ireland with severe OI who have received Palivizumab developed RSV bronchiolitis Nationally $17 \%$ of non-prophylaxed infants with OI developed RSV positive bronchiolitis among the 121 admissions. Increased LOS, bed days consumption and PICU admission were observed among those with dual pathology of severe OI and RSV infection. We could not establish statistical significance for observations.

Conclusion Infants with severe forms of Osteogenesis Imperfecta seems to benefit from RSV immunoprophylaxis with Palivizumab. 